\title{
[67Ga] Ga-citrate and COVID-19-associated pneumonia: an unexpected absence of uptake
}

\author{
Edgar Zamora ${ }^{1}$ (D) - Ana Y. Valdivia ${ }^{1} \cdot$ Benjamin Zalta ${ }^{2} \cdot$ Lionel S. Zuckier $^{1}$
}

Received: 4 May 2020 / Accepted: 21 May 2020 / Published online: 13 June 2020

(C) Springer-Verlag GmbH Germany, part of Springer Nature 2020

An elderly diabetic woman, presented from an outside institution with acute respiratory failure, complaining of headaches, fevers, and cough over several days. She was mildly neutropenic on admission with a slightly elevated ferritin level. The chest radiograph showed bilateral patchy opacities, and SARS-CoV-2 infection was confirmed by reverse transcription polymerase chain reaction. The clinical presentation also included the suggestion of right-sided mastoiditis supported by CT findings; she did not improve on aggressive antibiotic therapy with worsening leukocytosis and rising ferritin levels. Additional medications included apixaban, steroids, and insulin. Bone and gallium scintigraphy were requested to evaluate the extent of osseous and soft-tissue infection at the skull base [1].

${ }^{99 \mathrm{~m}} \mathrm{Tc}-\mathrm{MDP}$ bone scan demonstrated increased right mastoid uptake; gallium scintigraphy performed at $48 \mathrm{~h}$ demonstrated overall normal biodistribution with focal intense mastoid uptake (planar, top transaxial images). Extensive typical COVID-19-related lung infiltrates were noted on SPECT/CT;

This article is part of the Topical Collection on Image of the month

Human Research Ethics Committee (HREC) is not required for case reports in the USA nor at the authors' institution. Patient's age and other identifying features have been redacted to protect patient anonymity.

Edgar Zamora

zamoramed@gmail.com

1 Division of Nuclear Medicine, Department of Radiology, Montefiore Medical Center and the Albert Einstein College of Medicine, 1695A Eastchester Road, Bronx, NY, 10461 USA

2 Division of Cardiothoracic Imaging, Department of Radiology, Montefiore Medical Center and the Albert Einstein College of Medicine, 111 East 210th Street, Bronx, NY, 10467-2401 USA more diffuse than on prior bone scintigraphy but associated with very minimal gallium activity (planar, lower transaxial images). The patient succumbed from COVID-19 complications 11 days following the gallium imaging.

The finding of worsening COVID-19-related pneumonia with negligible gallium uptake contrasts with our observation of gallium's effectiveness to identify mastoid bone suppuration and may relate to differences in pathogen (virus vs. bacteria), substrate (lung vs. bone), or nature of pathology (suppurative vs. ARDS-like process). Minimal lung uptake also strikingly differs from the relatively intense lung uptake reported with FDG [2-4]. Further exploration of differences in the molecular basis of gallium and FDG localization, and variation in the pathophysiology of mastoiditis and COVID-19related lung pathology, will help shed light on these observations. From a clinical perspective, while gallium scintigraphy is often regarded as a generally sensitive tool to identify infectious processes in the lungs [5], it appears that COVID-19related lung lesions may not be included in this blanket generalization. 


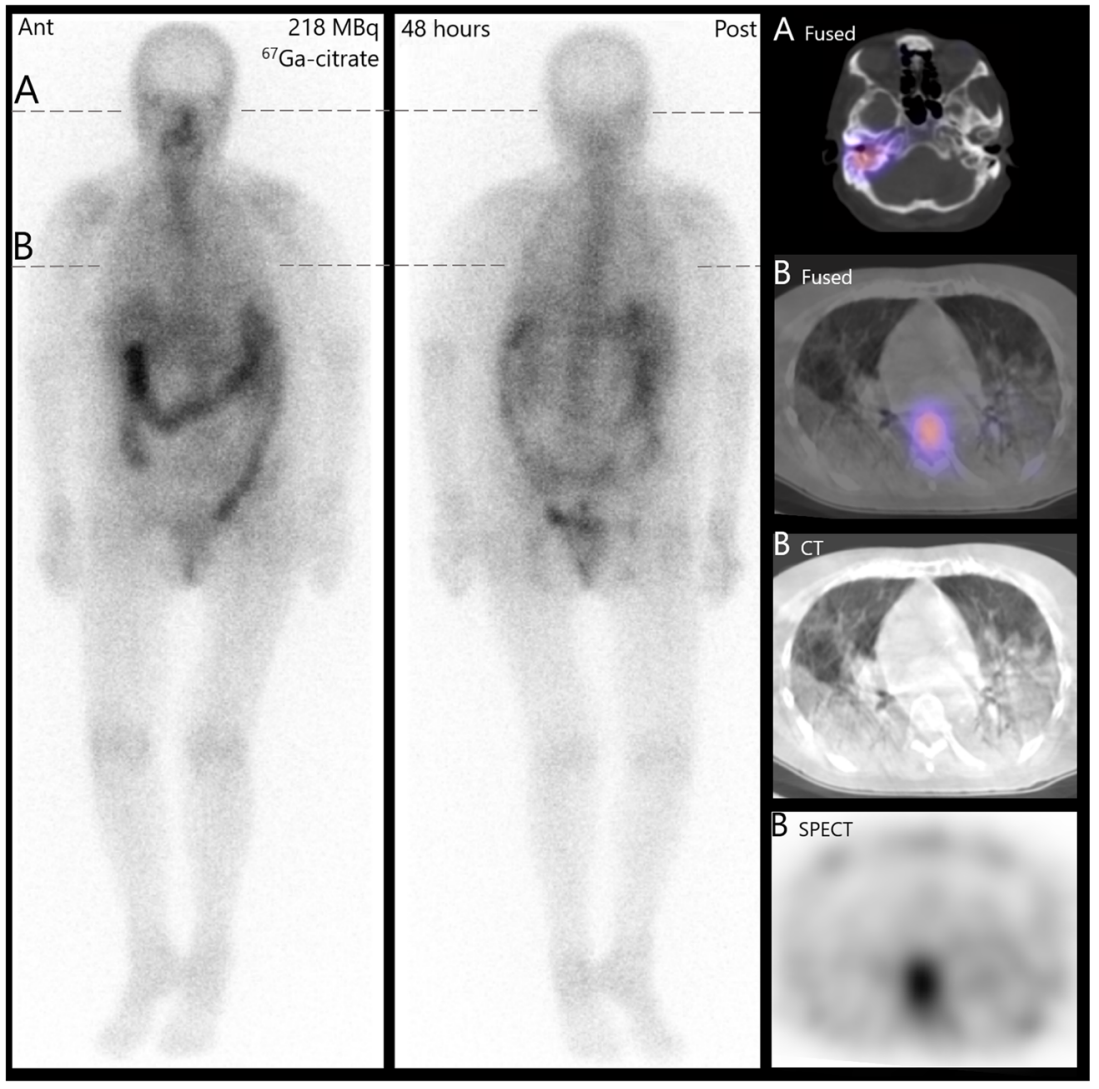

\section{Compliance with ethical standards}

Conflict of interest The authors declare that they have no conflicts of interest and have not received any type of funding related to this work.

Ethics approval All procedures performed in studies involving human participants were in accordance with the ethical standards of the institutional research committee and with the 1964 Helsinki declaration and its later amendments or comparable ethical standards. No studies involving animals are described in this article.

Patient Consent Patient identity in this retrospective single case report has been fully anonymized to comply with privacy standards.

\section{References}

1. Strashun AM, Nejatheim M, Goldsmith SJ. Malignant external otitis: early scintigraphic detection. Radiology. 1984;150(2):541-5.
2. Qin C, Liu F, Yen TC, Lan X. (18)F-FDG PET/CT findings of COVID-19: a series of four highly suspected cases. Eur J Nucl Med Mol Imaging. 2020;47(5):1281-6. https://doi.org/10.1007/ s00259-020-04734-w.

3. Zou S, Zhu X. FDG PET/CT of COVID-19. Radiology. 2020;200770. https://doi.org/10.1148/radiol.2020200770.

4. Setti L, Kirienko M, Dalto SC, Bonacina M, Bombardieri E. FDG$\mathrm{PET} / \mathrm{CT}$ findings highly suspicious for COVID-19 in an Italian case series of asymptomatic patients. Eur J Nucl Med Mol Imaging. 2020. https://doi.org/10.1007/s00259-020-04819-6.

5. Schuster DM, Alazraki N. Gallium and other agents in diseases of the lung. Semin Nucl Med. 2002;32(3):193-211. https://doi.org/10. 1053/snuc.2002.124178.

Publisher's note Springer Nature remains neutral with regard to jurisdictional claims in published maps and institutional affiliations. 